\title{
Some aspects of induced resistance to rice blast fungus, Magnaporthe grisea, in rice plant infested by white-backed planthopper, Sogatella furcifera
}

\author{
Hiroo Kanno, ${ }^{1, *}$ Masaru SAToh, ${ }^{1}$ Takashi Kimura ${ }^{1}$ and Yoshikatsu FuJita ${ }^{2}$ \\ ${ }^{1}$ Kyushu Okinawa National Agricultural Research Center; Kumamoto 861-1192, Japan \\ ${ }^{2}$ Hokkaido National Agricultural Research Center; Sapporo 062-8555, Japan \\ (Received 21 May 2004; Accepted 15 September 2004)
}

\begin{abstract}
The effect of previous rice plant infestation of the white-backed planthopper, Sogatella furcifera, on the incidence of rice blast disease caused by the fungal pathogen, Magnaporthe grisea, was investigated. The fungal pathogen $M$. grisea was less likely to cause symptoms of leaf blast on rice plants that had been previously infested with $S$. furcifera than on uninfested plants. This indicates that resistance to $M$. grisea was induced in rice plants by infestation of $S$. furcifera. The number of blast lesions per plant decreased with increasing numbers of infesting S. furcifera from 0 to 10 pairs. The active period of induced resistance was maintained for a relatively long time being greater than $15 \mathrm{~d}$. The difference in the incidence of leaf blast between $S$. furcifera infested and uninfested plants in the booting stage became smaller than that in the tillering stage, although there was a still significant difference in blast incidence between both infested and uninfested plants in booting stage. There was no significant difference in the incidence of leaf blast between damaged plants treated with needling and untreated control plants. This suggests that the simple mechanical effect to the plants caused by stylet insertion (penetration) and subsequent probing with the feeding behavior of $S$. furcifera was not related to the observed phenomenon. In S. furcifera infested plants, the expression of two genes regarding $\beta$-1,3-glucanase, Gns 4 and Gns5, was confirmed by RT-PCR analysis. These results show that infestation of $S$. furcifera apparently induced physiological changes including gene expression that were related to pathogen resistance to M. grisea in rice plants.
\end{abstract}

Key words: Induced resistance; rice; Sogatella furcifera; Magnaporthe grisea; $\beta$-1,3-glucanase

\section{INTRODUCTION}

Many of the studies on herbivorous insects and also on pathogens have been conducted to clarify the relationship of insects to plants and of pathogens to plants. However, studies on the relationship between insects and pathogens through host plants have seldom been conducted (Karban et al., 1987; Kanno and Fujita, 2003).

The white-backed planthopper, Sogatella furcifera, and the rice blast disease caused by Magnaporthe grisea, are economically important insect pest and disease respectively, of rice, Oryza sativa, throughout Southeast and Far-East Asia, including Japan. In general, S. furcifera does not hibernate in Japan. All of the original S. furcifera population emigrates from Mainland China into Japan during the rainy season from early June to early July. Then the population of the next generation of S. furcifera increases rapidly in rice fields, and the highest peak of the population appears at around late July or early August (Watanabe et al., 1991; Watanabe, 1996). At the same time, the rice blast disease caused by $M$. grisea also commonly develops in rice fields in Japan, especially those in the Kyushu area, and occasionally causes serious damage to the rice plants (Iwano, 1999). The authors, therefore, were interested in the interspecific relationships between $S$. furcifera and $M$. grisea through the host plants, and recently found that the resistance to the rice blast disease caused by $M$. grisea in the rice is induced by previous infestation of $S$. furcifera. Furthermore, it was confirmed that the induced resistance in the rice is systemic and is caused by the feeding behavior of $S$. furcifera (Kanno and Fujita, 2003). In this paper, the results

* To whom correspondence should be addressed at: E-mail: hkanno@affrc.go.jp DOI: 10.1303/aez.2005.91 
of studies on some other important aspects of this phenomenon are introduced and discussed.

\section{MATERIALS AND METHODS}

Test plants. Japonica rice variety Hinohikari, containing blast-resistant genes $\mathrm{Pi}-\mathrm{a}$ and $\mathrm{Pi}-\mathrm{i}$ (Iwano, 1999), was used in all experiments. Plants were grown under glasshouse conditions $\left(25 \pm 1^{\circ} \mathrm{C}\right.$, $60-80 \% \mathrm{RH}$ ) from seeds planted in plastic pots containing a commercial potting soil, Hinokunishodo (Japan Agricultural Co.), mixed with fertilizers (nitrogen: $0.02 \%$, phosphoric acid: $0.05 \%$, potassium: $0.04 \%$ ). Tests were conducted on plants grown to around the 5-leaf-stage, about 4 weeks post seeding.

Test insects. $S$. furcifera was from a laboratoryreared culture originating from adults collected in 1990 from a rice field in Chikugo, Fukuoka Prefecture, Japan. The susceptible Japonica rice variety "Reihoh" to $S$. furcifera was used for insect rearing. Adults of $S$. furcifera were allowed to oviposit on rice plants grown to the 2-leaf-stage in an insect rearing cage $(25 \mathrm{~cm} \times 30 \mathrm{~cm} \times 25 \mathrm{~cm})$. The rearing cages containing approximately 1,000 planthoppers individually were put in a controlled environment chamber $\left(25^{\circ} \mathrm{C}, \quad 50-70 \% \mathrm{RH}, \mathrm{L} 14: \mathrm{D} 10\right)$ until adults emerged $20 \mathrm{~d}$ after oviposition. These adults were used for all experiments.

Test fungal inoculums. The colony of $M$. grisea (race number: 007) used for this experiment was established in 1995 from a diseased rice paddy in Nishigoshi, Kumamoto Prefecture, Japan. The colony was incubated on oatmeal sucrose agar (OMSA) at $25^{\circ} \mathrm{C}$ in the dark for 2 weeks. The OMSA plates were then flooded with a $0.02 \%$ Tween 20 (surfactant) solution. The conidial suspension of $M$. grisea was prepared by softly scraping the mycelia from the surface of the plates using a paintbrush. A suitable concentration of conidia in suspension (about 500 per $\mathrm{mm}^{2}$ ) was carefully determined by microscopic observation at a magnification of $\times 150$, by adding distilled water.

Test 1: Effect of number of infested $S$. furcifera on incidence of rice blast disease caused by $M$. grisea. Test 1 was conducted to verify the effect of the number of infested S. furcifera on the incidence of rice blast disease caused by $M$. grisea. Forty rice plants at the 5-leaf-stage covered with transparent plastic cylinders $(15 \mathrm{~cm}$ in diameter and $70 \mathrm{~cm}$ in height) were divided into four equal groups. Each group of 10 plants was infested with a different number of $S$. furcifera, specifically 0,2 , 5 or 10 pairs of adults per plant. The first, 0 , category was the uninfested control. These adults were released into a cylinder, and allowed to feed and oviposit on the plant. Two days later, all treated and control plants were removed from the cylinders, and sprayed with $2 \mathrm{ml} /$ plant of conidial suspension using an airbrush sprayer (PIECE COM: PCWIDE 308). Plants were incubated in a dew chamber $\left(25^{\circ} \mathrm{C}, 100 \% \mathrm{RH}\right)$ for $18 \mathrm{~h}$ before being transferred to the glasshouse. The number of typical blast lesions, called S-lesion (susceptible lesion), on the plants was counted and compared among the treatments at $7 \mathrm{~d}$ post inoculation. The experiment was replicated three times. The data were analyzed by Tukey-Kramer test (SAS, 2000).

Test 2: The stability of induced resistance over time. Test 2 was designed to investigate the stability of the induced resistance in rice over time. Fifty rice plants at the 4-leaf-stage were prepared for the test, and divided into five equal groups. Groups of 10 plants each were assigned to one of five categories, $0,5,10$ and $15 \mathrm{~d}$ after $S$. furcifera infestation for $48 \mathrm{~h}$ and another group for the uninfested control. Firstly, the ten rice plants of the $15 \mathrm{~d}$ group were set in a cage $(50 \times 50 \times 50 \mathrm{~cm}$, plastic rod frame covered with a fitted cotton mesh net), then 100 pairs of newly emerged $S$. furcifera adults were released into the cage, and allowed to feed and oviposit on the plant. Two days later, the test plants were removed from the cage and put in the glasshouse condition. The other three groups, 10, 5 and $0 \mathrm{~d}$, received the same treatment every $5 \mathrm{~d}$. The 10 plants in the uninfested control group were placed in a second cage for $48 \mathrm{~h}$ with no additional insects, when the $0 \mathrm{~d}$ group was treated. Then, all of the test plants were inoculated with $M$. grisea and incubated as described in Test 1 . After $7 \mathrm{~d}$, the number of S-lesions of rice blast on the plants was counted. The experiment was replicated two times. The data were analyzed by Tukey-Kramer test (SAS, 2000).

Test 3: Effect of plant stage on induced resistance. Test 3 was conducted to confirm the effect of plant stage on induced resistance. Twenty rice plants at the 4-leaf-stage (tillering stage: vegetative stage) and another 20 plants at the 8-leaf-stage (booting stage: reproductive stage) were prepared 
and used in this experiment. The test plants in both leaf stages were divided into two groups, $S$. furcifera infested group and uninfested control group. The groups infested with $S$. furcifera at both leaf stages containing 10 plants each were put in the cage mentioned above, then 100 pairs of newly emerged $S$. furcifera adults were released into the cage, and allowed to feed and oviposit on the plant. The other two groups consisting of the uninfested category in both leaf stages were also placed in two other cages (same size, but no insects) for use as control plants. Two days later, these infested and uninfested plants were removed from the cages and then inoculated with $M$. grisea by the same method described above. We counted the number of S-lesions on the plants at $7 \mathrm{~d}$ after inoculation. The experiment was replicated two times. The data were analyzed by Tukey-Kramer test (SAS, 2000).

Test 4: Effect of mechanical damage on the incidence of rice blast disease caused by $M$. grisea. This test was conducted to clarify the effect of mechanically simulating $S$. furcifera feeding on induced resistance to $M$. grisea. Twenty rice plants at the 5-leaf-stage were prepared and divided into two groups for the test. The leaves of one of the groups of 10 plants were mechanically damaged by puncture using a sewing needle. The plant was punctured 50 times every $12 \mathrm{~h}$ for $48 \mathrm{~h}$, so that the total number of needle punctures was 250 per plant. Another group of 10 plants was used as the untreated control. The test plants were then inoculated with $M$. grisea. Inoculation, incubation and investigation were conducted by the same methods described above. The test was replicated twice. The data obtained was analyzed by Tukey-Kramer test (SAS, 2000)

Test 5: Genes expression coding PR-protein, $\beta$-1,3-glucanase, in rice plant infested by $S$. furcifera. This test was conducted to investigate the gene expression coding for one of the PR-proteins, $\beta$-1,3-glucanase, in rice plants infested by $S$. furcifera. Sixty rice plants at the 5-leaf-stage were prepared and divided into two groups, S. furcifera infested and uninfested plants. In this test, $S$. furcifera infestation was restricted to the stems of the test plants, which were encased in transparent plastic cylinders $(5 \mathrm{~cm}$ in diameter and $15 \mathrm{~cm}$ in height). Ten pairs of $S$. furcifera were released into each cylinder in the infested group. Twenty four hours later, the leaves of all test plants were cut off from the stems using scissors, and immediately frozen in liquid nitrogen and stored at $-80^{\circ} \mathrm{C}$. For isolation of total RNA, $2 \mathrm{~g}$ of frozen material were ground in liquid nitrogen and resuspended in $20 \mathrm{ml}$ of extraction butter [ $2 \%$ cetyltrimethylammonium bromide, $0.1 \mathrm{M}$ Tris- $\mathrm{HCl}$ (pH 9.5), $20 \mathrm{~mm}$ EDTA, $1.4 \mathrm{M} \mathrm{NaCl}, 1 \% \beta$-mercaptoethanol] and incubated at $65^{\circ} \mathrm{C}$ for $10 \mathrm{~min}$. The aqueous phase was extracted twice with an equal volume of chloroform/isoamyl alcohol (24:1). $\mathrm{LiCl}$ precipitation with $1 / 4$ volume $10 \mathrm{M} \mathrm{LiCl}$ was repeated twice, followed by resuspension in DEPC-treated water. The mRNA was isolated from the total RNA using an Oligotex-dT30 mRNA Purification Kit (TaKaRa). For RT-PCR analysis, cDNA was synthesized from $1 \mu \mathrm{g}$ of mRNA using a First-strand cDNA Synthesis Kit (TaKaRa). One or $4 \mu 1$ of the diluted cDNA (1/230) was used for analysis of a rice actin mRNA or two types of $\beta$-1,3-glucanase mRNAs respectively. The expression of a rice actin gene (GenBank accession number X16280 and AC091532) was used as the control. The amplification conditions were as follows: $2 \mathrm{~min}$ at $94^{\circ} \mathrm{C} ; 32$ cycles of $30 \mathrm{~s}$ at $94^{\circ} \mathrm{C}, 30 \mathrm{~s}$ at $55^{\circ} \mathrm{C}, 1 \mathrm{~min}$ at $68^{\circ} \mathrm{C}$. Primer pairs were as follows: Gns4, 5'-gctctgatcattggagcatttgc and 5 '-tggegtagttgaggetgatgtcg; Gns5, 5'-ttggagttgcggccattcctac and 5'-gagcgcgtagttgatgtcgatc; actin gene, 5'-tcaagtaccccatcgagcatgg and $5^{\prime}$-caatccacatctgctggaatgt.

\section{RESULTS}

Effect of number of infested $S$. furcifera on incidence of rice blast disease caused by $M$. grisea

The symptom of rice blast disease initially appears as lesions on the rice leaves that are confirmed with the naked eye on the rice leaves from around $4 \mathrm{~d}$ after inoculation of the conidial suspension of M. grisea in the glasshouse condition, $25^{\circ} \mathrm{C}$ and $70-80 \% \mathrm{RH}$. The number of S-lesion indicating typical symptoms of rice blast disease differed significantly between $S$. furcifera infested rice and uninfested rice. In the infested rice group, S-lesions were seldom found on the plant, although many S-lesions were observed on uninfested control plants. A decreasing tendency in the number of S-lesions per plant was apparently observed with increasing numbers of infested insects, and the difference among the treatments was significant $(p<0.05)$, as shown in Fig. 1. The incidence of leaf 


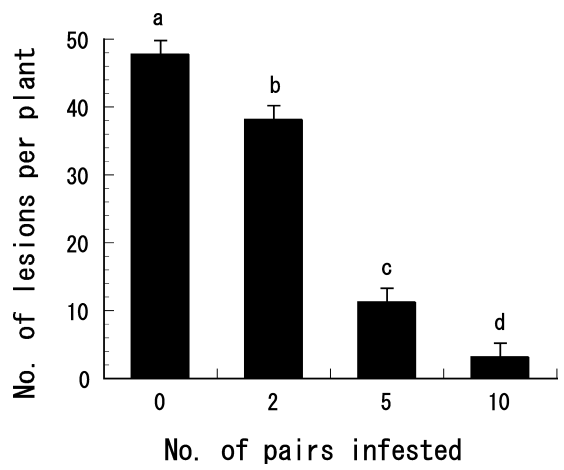

Fig. 1. Incidence of rice blast disease caused by Magnaporthe grisea on rice (Japonica variety: Hinohikari) previously infested by different numbers of the white-backed planthopper, Sogatella furcifera. Standard bars (SB) indicate mean number of blast lesions per plant, and vertical bars on the SB indicate standard errors. Means accompanied by different letters are significantly different at $p<0.05$ (Tukey-Kramer test; SAS, 2000).

blast in rice infested by $S$. furcifera was significantly lower than that in uninfested control plants, even with the low density infestation of only two pairs of $S$. furcifera.

\section{The stability of induced resistance over time}

The number of S-lesions on S. furcifera infested plants $1 \mathrm{~d}$ after infestation was quite small compared with that on uninfested control plants. The lesions gradually increased over time from 0 to 15 $\mathrm{d}$ after infestation. However, the number of lesions on plants infested by $S$. furcifera was still significantly smaller $(p<0.05)$ than that on uninfested control plants, even at $15 \mathrm{~d}$ after infestation, as shown in Fig. 2.

\section{Effect of plant stage on induced resistance}

The incidence of rice blast varied greatly according to the plant stage. In the case of plants at the tillering stage (vegetative stage) 4 weeks after planting, the difference in lesion numbers between $S$. furcifera infested and uninfested plants was highly significant $(p<0.05)$. On the other hand, in the case of plants at the booting stage (reproductive stage) 8 weeks after planting, the incidence of leaf blast was apparently lower than that in the tillering stage in both $S$. furcifera infested and uninfested control plants, as shown in Fig. 3. The difference of incidence of leaf blast between S. furcifera infested and uninfested plants was much smaller than that in the tillering stage, although the difference

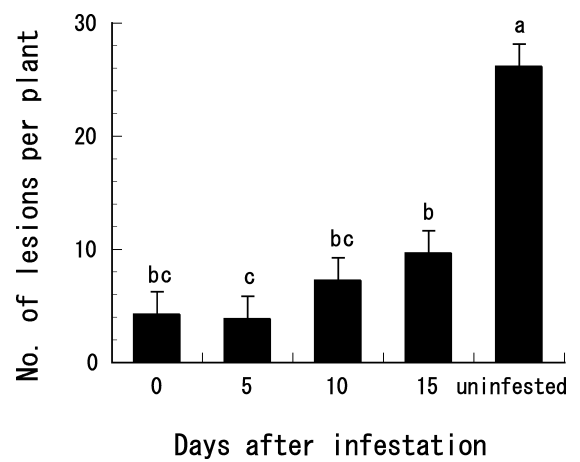

Fig. 2. Effect of the dates elapsed after infestation of Sogatella furcifera on incidence of rice blast disease caused by Magnaporthe grisea on rice (Japonica variety: Hinohikari). Standard bars (SB) indicate mean number of blast lesions per plant, and vertical bars on the SB indicate standard errors. Means accompanied by different letters are significantly different at $p<0.05$ (Tukey-Kramer test; SAS, 2000).

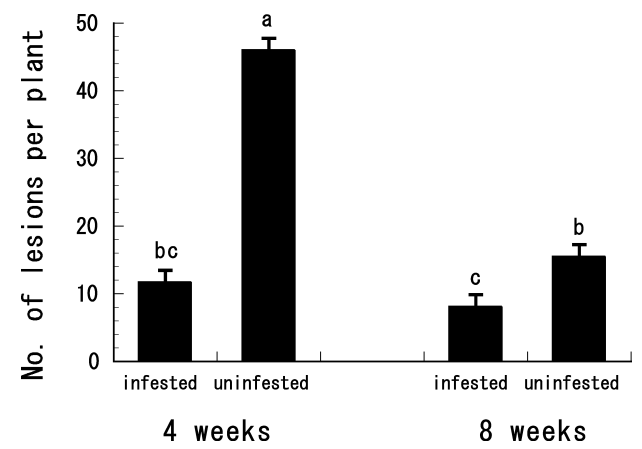

Fig. 3. Effect of plant stages, such as tillering stage and booting stage, on incidence of rice blast disease caused by Magnaporthe grisea on rice (Japonica variety: Hinohikari) previously infested by the white-backed planthopper, Sogatella furcifera. Standard bars (SB) indicate mean number of blast lesions per plant, and vertical bars on the SB indicate standard errors. Means accompanied by different letters are significantly different at $p<0.05$ (Tukey-Kramer test; SAS, 2000).

between the two groups was still significant.

\section{Effect of mechanical damage on the incidence of rice blast disease caused by $M$. grisea}

There was no significant difference $(p<0.05)$ in the incidence of leaf blast between damaged plants treated with needling and untreated control plants, although the mean value of the S-lesions in the plants treated with needling was slightly lower than that in untreated control plants, as shown in Fig. 4.

\section{Expression of $\beta$-1,3-glucanase genes in plants} infested by $S$. furcifera

The $\beta$-1,3-glucanase is well-known as a defense- 


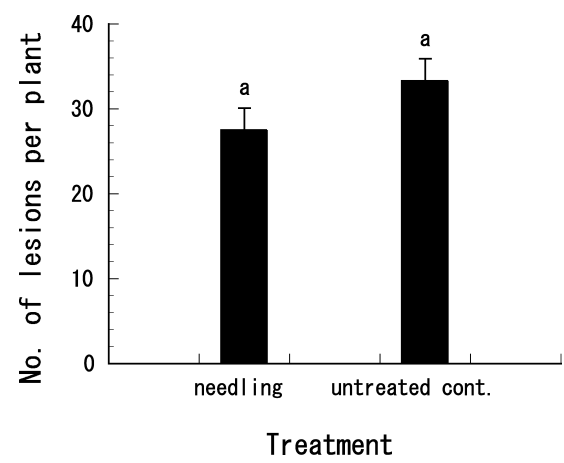

Fig. 4. Effect of mechanical wounding by needling on the incidence of blast disease caused by Magnaporthe grisea on rice (Japonica variety: Hinohikari). Standard bars (SB) indicate mean number of blast lesions per plant, and vertical bars on the SB indicate standard errors. Means accompanied by different letters are significantly different at $p<0.05$ (TukeyKramer test; SAS, 2000).

related gene induced in plants against fungi. RTPCR method was applied to detect the expression of $\beta$-1,3-glucanase genes, Gns 4 and Gns 5 (Romero et al., 1998). Amplification of genomic DNA was distinguishable from that of cDNA because of the presence of an intron. No contamination of genomic DNA occurred in the cDNA sample. Both genes were more expressed in S. furcifera infested plants compared with uninfested control plants. In particular, the induction of Gns5 expression was clearly significant, as shown in Fig. 5.

\section{DISCUSSION}

The interspecific relationship between two kinds of insects has been studied in several species. Attacks by herbivorous insects induce chemical and physical changes in many host plants (Green and Ryan, 1972; Tallamy and Raupp, 1991; Baldwin, 1994; Dicke, 1994; Schoonhoven et al., 1998). In several systems host changes caused by herbivore damage have had deleterious effects on subsequent herbivores. Herbivores that fed on damaged plant tissue had lower survival rates, reduced individual growth rates, and reduced adult weight or fecundity, or both (Karban and Myers, 1989; Denno et al., 1995). However, the interspecific relationship between insects and pathogens has seldom been studied (Karban et al., 1987; Kanno and Fujita, 2003). The negative interspecific effects between an herbivorous insect and a fungus were first observed in both the spider mite, Tetranychus urticae,
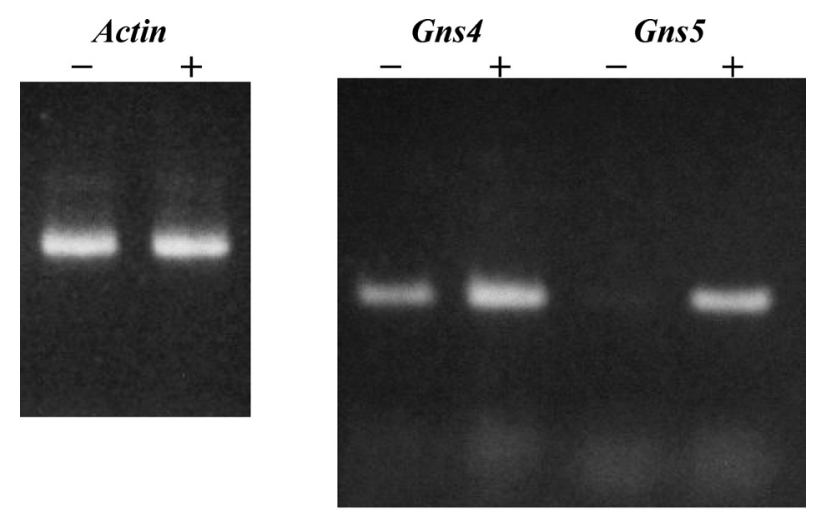

$$
\begin{aligned}
& +: \text { S. furcifera infested plant } \\
& -: \text { uninfested control plant }
\end{aligned}
$$

Fig. 5. Effect of $S$. furcifera infestation on the expression of $\beta$-1,3-glucanase genes. Two genes, Gns 4 and Gns5, were investigated by RT-PCR analysis. The expression of a rice actin gene was used as an internal control.

and the fungal pathogen, Verticillium dahliae. It was reported that changes in cotton seedlings caused by previous exposure to spider mites reduced the probability of infection and severity of symptoms caused by the vascular wilt fungus, $V$. dahliae (Karban et al., 1987).

Kanno and Fujita (2003) recently demonstrated the following phenomenon in rice plants. Rice plants that had previously been infested with $S$. furcifera were less likely than controls to develop the blast disease caused by $M$. grisea. The number of blast lesions, especially S-lesions, on the leaves of rice plants that were infested with $S$. furcifera was significantly lower than that on the uninfested plants. Further, the blast incidence was strongly suppressed by infestation with either male or female adults of $S$. furcifera, and no significant difference between the two sexes was observed. There was also a significant difference in the number of lesions between $S$. furcifera infested and uninfested plants when the infestation was restricted only to the rice plant stems. In this case, the lesion number on the plants that were previously infested with $S$. furcifera was around $40 \%$ lower than that of uninfested control plants.

The results of Kanno and Fujita (2003) show that $S$. furcifera infestation can strongly inhibit the development of rice blast symptoms. S. furcifera was no longer present in the first experiment when rice plants were inoculated with conidia, therefore infestation of $S$. furcifera must have induced physi- 
ological changes in the plant that reduced its susceptibility to the fungus, $M$. grisea. The incidence of blast disease did not significantly differ in the presence of either all-male or all-female $S$. furcifera, thus they hypothesize that the lower disease incidence was induced by the feeding behavior of either sex rather than the oviposition behavior of females. The incidence of rice blast is also suppressed by restricted infestation of $S$. furcifera to only the stems of the host plant. This result indicates that induced physiological changes in the plants, reducing susceptibility to $M$. grisea, are a systemic phenomenon.

We also report here on the induced systemic resistance in rice to $M$. grisea, the causal agent of the rice leaf blast. The results of our present experiment basically support those of the studies described above, and more detailed phenomena were further elucidated. The incidence of the leaf blast is negatively correlated with the number of $S$. furcifera infested on the plants, and the results indicate that strong resistance is induced in rice by $S$. furcifera infestation, because the lesion number on the plants infested by even two pairs (two males and two females) was significantly smaller than that in uninfested control plants. This induced resistance continues for a relatively long time of more than $15 \mathrm{~d}$, and is strongly enhanced in the vegetative stage compared to the reproductive stage of the plants. An important implication of this result is that the susceptibility to $M$. grisea in rice must be reduced with growth of the plant from the vegetative stage to reproductive stage. Furthermore, the blast incidence was not affected by mechanical wounding. This result suggests that the simple mechanical effect to the plants caused by stylet insertion (penetration) and subsequent probing with feeding behavior of $S$. furcifera is not related to the phenomenon.

Several possible mechanisms could produce a general resistance pattern. Resistance may result from a physiological change in the plant, which requires no mechanism of recognition or response to a unique component of the attacking pathogen. Such a physiological change need not be adaptive for the plant but may reflect wounding or deterioration of metabolic functions. The first species attacking the plant may deplete the most desirable nutrients or plant parts. Alternatively, resistance may be caused by metabolites that undergo activa- tion or de novo synthesis and accumulate in the plant after attack. Chemicals that accumulate after damage may have activity against a variety of organisms. For example, phytoalexin isoflavonoids reduce fungal growth and also deter feeding by herbivorous insects (Karban et al., 1987).

Recently, technological advances of molecular biology have clarified the mechanism of such induced resistance in plants at the molecular level. For example, in the tomato plant, it has been demonstrated that mechanical wounding, herbivore attack and pathogen attack can elicit a defense system, such as the jasmonic acid pathway, salicylic acid pathway, etc., and then some phytoalexins and pathogenesis related proteins (PR-proteins) against pathogens and herbivores are finally induced in the plants (Fidantsef et al., 1999; Sivasankar et al., 2000; Orozco-Cardenas et al., 2001). In rice plants, Schweizer et al. $(1997,1998)$ also confirmed that some PR-proteins are produced in the rice with activation of the jasmonic acid pathway by pathogen attack and also mechanical wounding. The authors also confirmed in this study that two genes, Gns4 and Gns5, coding for one of the PR-proteins, $\beta$ 1,3-glucanase, which indicates a strong antimicrobial activity, were expressed in rice plants infested by $S$. furcifera. We now speculate that these two genes may not have been expressed in rice plants by mechanical wounding with treatment of needling, and are presently trying to verify this.

The present results also indicate that some physiological changes may occur in rice plants fed on by $S$. furcifera. One of the possible mechanisms of the phenomenon is speculated to be as follows. 1) Saliva of $S$. furcifera contains an elicitor regarded as a trigger of the phenomenon; 2) in the rice plant fed on by $S$. furcifera, defense systems, such as the jasmonic acid pathway and the salicylic acid pathway, are activated by the elicitor; 3 ) then the phytoalexins and PR-proteins, not only $\beta$-1,3-glucanase but also others, that protect against pathogens are finally produced in the rice. This hypothesis regarding the mechanism will be investigated as the next step of this research series.

\section{ACKNOWLEDGEMENTS}

We thank Drs. K. Hirayae and T. Nakajima (Plant Pathology Lab., Kyushu Okinawa National Agricultural Research Center: KONARC) for their advice on experimental measures of the rice blast fungus, $M$. grisea, and Mrs. Hidaka (KONARC) for her assistance in preparation of $M$. grisea. We also thank two 
anonymous reviewers for their helpful comments on the manuscript.

\section{REFERENCES}

Baldwin, I. T. (1994) Chemical changes rapidly induced by folivory. In Insect-Plant Interactions. Vol. 5 (E. A. Bernays ed.). CRC Press, Boca Raton, FL, pp. 1-23.

Denno, R. F., M. S. McClure and J. R. Ott (1995) Interspecific interactions in phytophagous insects: Competition reexamined and resurrected. Ann. Rev. Entomol. 40: 297-331.

Dicke, M. (1994) Local and systemic production of volatile herbivore-induced terpenoids: Their role in plant-carnivore mutualism. J. Plant Physiol. 143: 465-472.

Fidantsef, A. L., M. J. Stout, J. S. Thaler, S. S. Duffey and R. M. Bostock (1999) Signal interactions in pathogen and insect attack: expression of lipoxygenase, protainase inhibitor II, and pathogenesis-related protein $\mathrm{P} 4$ in the tomato, Lycopersicon esculentum. Physiol. Molec. Plant Pathol. 54: 97-114.

Green, T. R. and C. A. Ryan (1972) Wound-induced proteinase inhibitor in plant leaves: A possible defense mechanism against insects. Science 175: 776-777.

Iwano, M. (1999) Presumed genotypes for true resistance of recommended rice varieties to rice blast in Kyushu Okinawa districts. Kyushu Agric. Res. 64: 69.

Kanno, H. and Y. Fujita (2003) Induced systemic resistance to rice blast fungus in rice plants infested by whitebacked planthopper. Entomol. Exp. Appl. 107: 155-158.

Karban, R., R. Adamchak and W. C. Schnathorst (1987) Induced resistance and interspecific competition between spider mites and a vascular wilt fungus. Science 235: 678-680.

Karban, R. and Myers, J. H. (1989) Induced plant responses to herbivory. Ann. Rev. Ecol. System. 20: 331-348.

Orozco-Cardenas, M. L., J. Narvaez-Vasquez and C. A. Ryan (2001) Hydrogen peroxide acts as a second messenger for the induction of defense genes in tomato plants in response to wounding, systemin, and methyl jasmonate. Plant Cell 13: 179-191.

Romero, G. O., C. Simmons, M. Yaneshita, M. Doan, B. R. Thomas and R. L. Rodoriguez (1998) Characterization of rice endo- $\beta$-glucanase genes (Gns2-Gns14) defines a new subgroup within the gene family. Gene 223: 311320 .

SAS Institute (2000) JMP, Version 4.0. SAS Institute Inc., Cary, North Carolina.

Schoonhoven, L. M., T. Jermy and J. J. A. van Loon (1998) Insect-Plant Biology. Chapman \& Hall, London. 409 pp.

Schweizer, P., A. Buchalas, P. Silverman, M. Seskar, I. Raskin and J.-P. Metraux (1997) Jasmonate inducible genes are activated in rice by pathogen attack without a concomitant increase in endogeneous jasmonic acid levels. Plant Physiol. 114: 79-88.

Schweizer, P., A. Buchalas, P. Silverman, M. Seskar, I. Raskin and J.-P. Metraux (1998) Induced systemic resistance in wounded rice plants. Plant J. 14: 475-481.

Sivasankar, S., B. Sherdrick and S. J. Rothstein (2000) Expression of allene oxide synthase determines defense gene activation in tomato. Plant Physiol. 122: 13351342.

Tallamy, D. W. and M. J. Raupp (1991) Phytochemical Induction by Herbivores. John Wiley, New York. 388 pp.

Watanabe, T. (1996) Population dynamics of long-range migratory rice planthoppers, Nilaparvata lugens and Sogatella furcifera, in Japan. Proceedings of the International Workshop on 'Pest management Strategies in Asian Monsooon Agroecosystems'. pp. 45-54.

Watanabe, T., K. Sogawa, Y. Hirai, M. Tsurumachi, S. Fukamachi and Y. Ogawa (1991) Correlation between migratory flight of rice planthoppers and the low-level jet stream in Kyushu, south-western Japan. Appl. Entomol. Zool. 26: 215-222. 